\title{
Drenaje subcutáneo por técnica de Beck para enfisema subcutáneo masivo en paciente pediátrico: reporte de caso
}

Pablo Mattos Navarro 1,a; Adela Magne 1,a; J. Shamir Barros-Sevillano ${ }^{2, b}$; Jim K. Gronerth-Silva 2,b; Jose CaballeroAlvarado $^{3, a}$; Joshuan J. Barboza-Meca* 4,c

\section{RESUMEN}

Diversas terapias son empleadas para el manejo del enfisema subcutáneo en adultos, pero no se ha encontrado alguna referencia del drenaje subcutáneo mediante la técnica de Beck en pacientes pediátricos. Presentamos el caso clínico de una paciente de catorce años de edad con un trauma cerrado debido a una caída desde una altura de diez metros. Al ingreso, la paciente muestra pérdida de conciencia y desarrolla un síndrome de distrés respiratorio, por lo que se le indica ventilación mecánica. Siete días después se produce un enfisema subcutáneo y se realiza una inserción con angiocatéter fenestrado empleando la técnica de Beck. El enfisema en la zona superior del tórax y el rostro disminuyó a las 24 horas, y a las 72 horas la resolución fue completa.

Palabras clave: Catéteres; Enfisema subcutáneo; Neumotórax; Adolescente (Fuente: DeCS BIREME).

\section{Subcutaneous drainage using Beck's technique for massive subcutaneous emphysema in a pediatric patient: a case report}

\section{ABSTRACT}

Several therapies are used for the management of subcutaneous emphysema in adults, but no reference to subcutaneous drainage using Beck's technique has been found in pediatric patients. This is the clinical case of a 14-year-old female patient who presented a blunt force trauma that resulted from a ten-meter-high fall. On admission, she was unconscious and developed respiratory distress syndrome, thus mechanical ventilation was indicated. Seven days later, subcutaneous emphysema occurred, and a fenestrated angiocatheter was inserted using Beck's method. At 24 hours, the emphysema was reduced in the upper chest and face, and, at 72 hours, complete resolution was observed.

Keywords: Catheters; Subcutaneous emphysema; Pneumothorax; Adolescent (Source: MeSH NLM).

1 Hospital Materno Infantil Caja Nacional de Salud. La Paz, Bolivia.

2 Universidad César Vallejo, Sociedad Científica de Estudiantes de Medicina (SOCIEM UCV). Trujillo, Perú.

3 Universidad Privada Antenor Orrego, Escuela de Posgrado. Trujillo, Perú.

4 Universidad Señor de Sipán, Escuela de Medicina. Chiclayo, Perú.

a Médico Cirujano.

b Estudiante de Medicina.

c Magister en Investigación Clínica.

* Autor corresponsal. 


\section{INTRODUCCIÓN}

El enfisema subcutáneo (ES) es una enfermedad ocasionada por la entrada de aire u otros gases en el interior de los tejidos blandos debajo de la piel ${ }^{(1)}$. Es un problema que se presenta con frecuencia luego de un traumatismo, una cirugía, los procesos invasivos en el tórax, un neumotórax traumático, o la ventilación mecánica, entre otros ${ }^{(2)}$.

Cerca del $1,3 \%$ de los casos puede complicarse y presentar deformidad abdominal, facial, genital y hasta comprensión de la tráquea y vasos sanguíneos, estos últimos comprometen gravemente las vías respiratorias, la circulación sanguínea hacia la cabeza y el retorno venoso ${ }^{(3)}$. Al mismo tiempo, la restricción de la reexpansión pulmonar completa puede conducir a una presión alta en las vías respiratorias, acidosis respiratoria severa, falla del ventilador, mal funcionamiento del marcapasos, compromiso de las vías respiratorias y fenómenos de tensión ${ }^{(4)}$.

Existen diversas terapias para el manejo del ES en adultos, como el corte supraclavicular (colocando un dren Penrose acompañado de leve frotación para que permanezca permeable), el drenaje por aspiración con diferentes catéteres (como los multiperforados) y el drenaje pasivo con angiocatéter de mínima medida reformada por la técnica de Beck ${ }^{(5-7)}$. Sin embargo, en los pacientes pediátricos aún no existen referencias del drenaje subcutáneo mediante la técnica de Beck ${ }^{(8)}$.
Reportamos el manejo de un caso de ES severo secundario a ventilación mecánica con la inserción de un angiocatéter fenestrado mediante la técnica de Beck, procedimiento que es utilizado por primera vez en una adolescente de 14 años de edad en nuestro medio.

\section{CASO CLÍNICO}

Paciente de sexo femenino de 14 años de edad, estudiante, originaria de Oruro, Bolivia, con un trauma cerrado posterior a una caída de una altura de diez metros. Ingresa con pérdida de conciencia. No había antecedentes importantes en la historia clínica. Luego del ingreso por urgencias, aparece el síndrome de distrés respiratorio y se indica ventilación mecánica. Los parámetros ventilatorios fueron VT $=530$; TI 1,2; PEEP = 7 y $\mathrm{FiO2}=7$, y los valores de gasometría mostraron los siguientes valores: $\mathrm{pH}=7,09 ; \mathrm{pO}=82 \mathrm{mmHg}$; pCO2 = 49; $\mathrm{HCO}=24 ;$ ExBase $-8 ;$ SatO2 $=72$. Pasados los siete días presenta un ES iatrogénico y recibe tratamiento conservador.

En las siguientes 48 horas, el enfisema se extiende progresivamente y compromete las extremidades superiores e inferiores, las zonas facial, torácica y genital; además, obstaculiza la apertura ocular y aparecen los problemas respiratorios. Debido a esto se procede a realizar la introducción del angiocatéter fenestrado mediante la técnica de Beck ${ }^{(8)}$ (Figura 1).

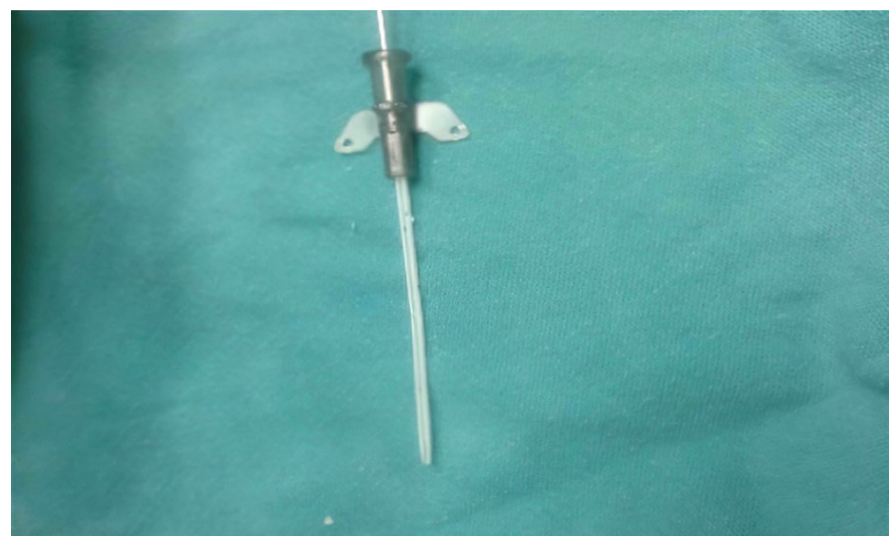

Figura 1. Catéter defenestrado

La técnica consistió en colocar dos de estos catéteres en el tejido celular subcutáneo a $2 \mathrm{~cm}$ de la línea media clavicular próximos a la superficie de la tercera costilla (Figura 2). Para ello, se realizó un corte aproximativo de dos centímetros para introducir el drenaje con dirección cefálica y luego la piel se cerró con un apósito (Figura 3). Se adapta la libación esporádica en un aspirador y maniobras de fricción leve en forma centrípeta y facilitar la aspiración. En este momento, se realizó una variación en la inserción del catéter con apoyo de una guía ecográfica para disminuir riesgos durante el procedimiento. 


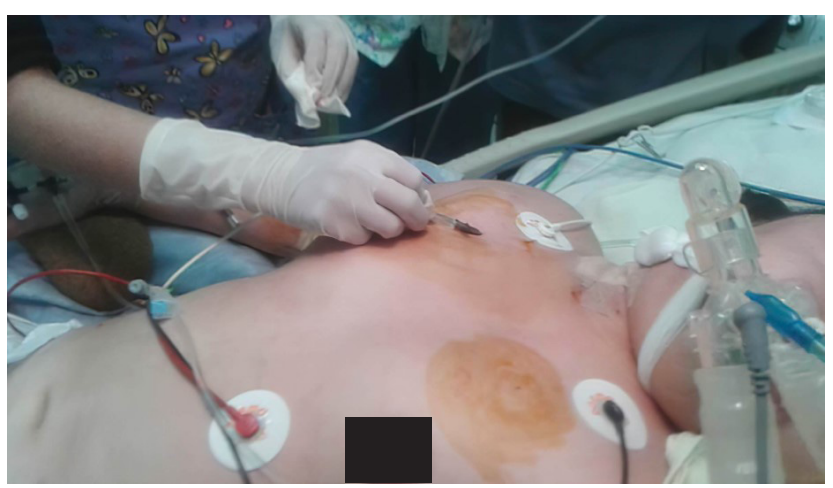

Figura 2. Localización del punto de inserción del catéter

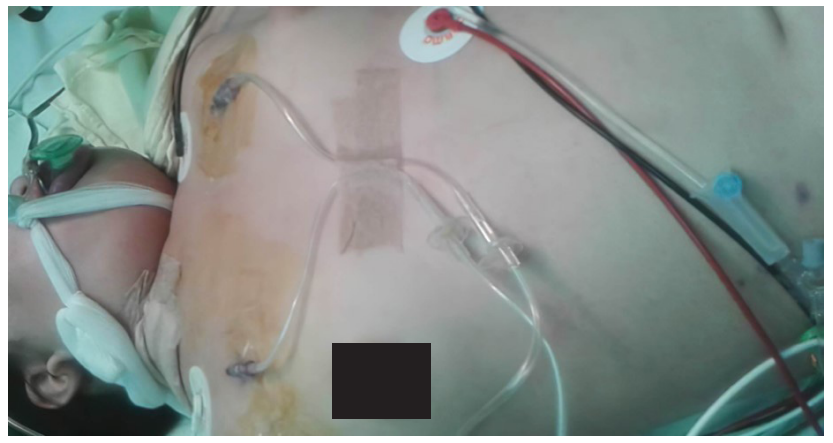

Figura 3. Inserción del catéter

Luego de 24 horas, la apertura ocular mejora y el ES se reduce en la en la zona torácica superior y el rostro. A las 72 horas, se observa la resolución completa del ES, y los resultados de la gasometría arterial se normalizan (parámetros ventilatorios: VT $=400 ;$ TI 1.2; $\mathrm{PEEP}=5$; $\mathrm{FiO}_{2}=5$ y valores de gasometría: $\mathrm{pH}=7,36 ; \mathrm{pO}_{2}=90$ $\mathrm{mmHg} ; \mathrm{pCO}_{2}=37 ; \mathrm{HCO}_{3}=21 ;$ ExBase $-4 ; \mathrm{SatO}_{2}=98 \%$.

\section{DISCUSIÓN}

Los probables mecanismos que influyen en el desarrollo del enfisema subcutáneo son diversos. Entre ellos están la lesión de la pleura parietal que permite el paso del aire a los tejidos pleurales y subcutáneos, el aire alveolar que extiende la vaina endovascular y el hilio pulmonar hacia la fascia endotorácica, el aire en el mediastino que se expande hacia las vísceras cervicales y otros planos de tejido conectados, aire procedente de fuentes externas y la generación local de gas causada por infecciones necrotizantes ${ }^{(2,4)}$.

La hinchazón alrededor del cuello acompañada de dolor en el pecho es el signo visible más común del ES. Otras manifestaciones incluyen el dolor de garganta, el dolor de cuello, la dificultad para tragar, disnea, sibilancias y distensión. Por lo tanto, el ES, a menudo, causa síntomas mínimos, no es peligroso en sí mismo y no requiere un tratamiento específico (2). Sin embargo, representa un dilema para el manejo. Para casos leves que no causan molestias significativas, la observación, tranquilidad ${ }^{(9)}$ y el manejo conservador son apropiados (10); incluso en casos de ventilación mecánica con presión positiva, se considera benigno y no siempre es necesario un ajuste de ventilación ${ }^{(11)} y$, rara vez, se tienen consecuencias fisiopatológicas. Cuando el cuadro es severo, muy incómodo, desfigurante o alarma al paciente y su familia, el médico puede sentirse en la obligación de tratarlo.

En la evolución de nuestra paciente observamos un ES extenso. Por ende, se procedió a intervenir utilizando la técnica descrita por Beck ${ }^{(8)}$, la cual se basa en hacer fenestraciones en forma de espiral a un angiocatéter tamaño 20G, de aproximadamente 2 a $3 \mathrm{~cm}$ laterales a la línea medioclavicular a nivel de la tercera costilla. Los catéteres se introducen en un ángulo de $45^{\circ}$ con una profundidad aproximada de 0,5 a $1 \mathrm{~cm}$ en la piel expandida por el ES, luego el ángulo de inserción disminuye y el catéter se dirige medialmente para que se inserte por completo y la punta tenga entre 1 a 1,5 cm de profundidad en la piel ${ }^{(8)}$.

Este drenaje subcutáneo es realizado en personas adultas sin las dificultades y complicaciones descritas hasta el momento, con una mejoría marcada a las 24 horas de la colocación ${ }^{(5,8)}$. En este caso se optó por esta técnica terapéutica por los siguientes beneficios: la simplicidad al colocar el equipo de aspiración y que no es necesario llevar al paciente al quirófano. El riesgo disminuyó al utilizar una guía ecográfica para insertar el catéter. Además, se reduce el peligro de una infección porque es un procedimiento estéril que se realiza en un sistema cerrado. Las complicaciones de la cicatrización también disminuyen porque es una técnica mínimamente invasiva y, por ende, la incomodidad del paciente es menor. La facilidad de construcción del catéter permite un tratamiento más temprano en su curso y evitar complicaciones graves ${ }^{(8)}$.

Si bien nuestra experiencia transcurrió sin problemas, existen dos posibles inconvenientes que podrían aparecer. El primero es la infección y el segundo es que el catéter puede bloquearse con sangre; sin embargo, puede reemplazarse fácilmente en un lugar diferente, por lo que se recomienda sustituir el catéter a la primera señal de complicaciones.

El empleo de la técnica de Beck en el tratamiento del ES pediátrico debe ser individualizado según la presentación y gravedad del cuadro clínico, aunque características como su alta efectividad, disponibilidad en la mayoría de las salas médicas, la mínima invasión, el bajo riesgo de infecciones, la sencillez del procedimiento y el bajo costo, lo sugieren como una excelente opción terapéutica.

En conclusión, el empleo de este procedimiento es inusual y 
Pablo Mattos Navarro; Adela Magne; J. Shamir Barros-Sevillano; Jim

K. Gronerth-Silva; Jose Caballero-Alvarado; Joshuan J. Barboza-Meca

hasta ahora no se encuentra evidencia científica suficiente de su ejecución en edad pediátrica. Sin embargo, en nuestro caso, esta técnica solucionó el enfisema subcutáneo en tres días, la paciente lo toleró fácilmente, ya que estaba sedada por la ventilación mecánica, y no necesitó de una intervención mayor.

Contribuciones de los autores: Pablo Mattos Navarro y Adela Magne: concepción y diseño del artículo, recolección de resultados, análisis e interpretación de datos, redacción del artículo, revisión crítica del artículo, aprobación de la versión final y aporte de pacientes o material de estudio. J. Shamir Barros-Sevillano, Jim K. Gronerth-Silva, José Caballero y Joshuan J. Barboza-Meca: concepción y diseño del artículo, análisis e interpretación de datos, redacción, revisión crítica del artículo, aprobación de la versión final e intervención de la paciente.

Fuentes de financiamiento: El artículo ha sido financiado por los autores.

Conflictos de interés: Los autores declaran que no existe ningún conflicto de intereses.

Exención de responsabilidad: Los autores confirman que el contenido del presente manuscrito es producto del escrutinio aplicado al participante y son responsabilidad estrictamente de los profesionales involucrados en la publicación de este artículo.

\section{REFERENCIAS BIBLIOGRÁFICAS}

1. Kaufman E, Leviner E, Galili D, Garfunkel AA. Subcutaneous air emphysema-a rare condition (four case reports). J Oral Med. 1984; 39(1): 47-50.

2. Maunder RJ, Pierson DJ, Hudson LD. Subcutaneous and mediastinal emphysema. Pathophysiology, diagnosis, and management. Arch Intern Med. 1984; 144(7): 1447-53.

3. Cesario A, Margaritora S, Porziella V, Granone P. Microdrainage via open technique in severe subcutaneous emphysema. Chest. 2003; 123(6): 2161-2.

4. Abu-Omar Y, Catarino PA. Progressive subcutaneous emphysema and respiratory arrest. J R Soc Med. 2002; 95(2): 90-1.

5. Aghajanzadeh M, Dehnadi A, Ebrahimi H, Fallah KM, Khajeh JS, Amir MA, et al. Classification and management of subcutaneous emphysema: a 10-year experience. Indian J Surg. 2015; 77(Suppl. 2): 673-7.

6. García MRM, Martín-López J, Arias-Díaz M, Díaz-Castellanos MA. Tratamiento del enfisema subcutáneo masivo con drenaje aspirativo. Med Intensiva. 2016; 40(4): 253-4.

7. Alarcón-Meregildo KG, Polo-Romero FJ, Beato-Pérez JL. Tratamiento de enfisema subcutáneo severo por microdrenaje. A propósito de un caso. Arch Bronconeumol. 2014; 50(1): 47-8.

8. Beck PL, Heitman SJ, Mody CH. Simple construction of a subcutaneous catheter for treatment of severe subcutaneous emphysema. Chest. 2002; $121(2):$ 647-9.

9. Jain P, Vanner T. Subcutaneous emphysema with pneumomediastinum during the second stage of labour: a rare intrapartum complication. J Gynecol Obstet. 2007; 9(1).

10. Rivares EJ, Gil PP, García MM. Tratamiento conservador del enfisema subcutáneo y neumomediastino. ORL Aragón. 2001; 4(2): 17-9.

11. Gries CJ, Pierson DJ. Tracheal rupture resulting in life-threatening subcutaneous emphysema. Respir Care. 2007; 52(2): 191-5.

Correspondencia:

Joshuan J. Barboza

Dirección: Calle Juan del Corral 937. Trujillo, Perú

Teléfono: +51992108520

Correo electrónico: jbarbozameca@relaped.com

Recibido: 01 de octubre de 2020.

Evaluado: 21 de diciembre de 2020.

Aprobado: 04 de enero de 2021.

( $)$ La revista. Publicado por Universidad de San Martín de Porres, Perú.

(c) $\mathbf{B r}$ Licencia de Creative Commons Artículo en acceso abierto bajo términos de Licencia Creative Commons Atribución 4.0 Internacional. (http://creativecommons.org/licenses/by/4.0/)

\section{ORCID iDs}

Pablo Mattos Navarro $\quad$ https: / / orcid.org/0000-0002-0724-5903

Adela Magne

J. Shamir Barros-Sevillano

(1) https: / / orcid.org/0000-0001-6535-8474

Jim K. Gronerth-Silva

https: / / orcid.org/0000-0003-2997-2092

Jose Caballero-Alvarado

(3) https: / / orcid.org/0000-0002-6401-9232

Joshuan J. Barboza

(1) https: / / orcid.org/0000-0002-2896-1407 